\title{
El viaje en la esfera pública europea. El caso del Programa Erasmus
}

\author{
Travel in the european public sphere. \\ The case of the Erasmus Programme
}

\author{
Cristina Fernández-Rovira \\ Universitat de Vic-Universitat Central de Catalunya \\ cristina.fernandez1@uvic.cat \\ Código Orcid: 0000-0003-0643-7329
}

\begin{abstract}
Resumen
El artículo aúna el viaje, la identidad y la esfera pública europea a través de la experiencia que ofrece el Programa Erasmus para los universitarios de la Unión Europea, lo que resulta de interés y novedoso para conocer de qué manera se interrelacionan los distintos conceptos en esta experiencia vital. Bajo un enfoque comparativo se describen las actitudes y percepciones de estudiantes españoles que han realizado un Erasmus y aquellos que no lo han hecho acerca del programa, de la construcción de la propia identidad europea y de la esfera pública común. El núcleo del análisis lo constituyen los datos obtenidos a través de cuestionario cerrado aplicado a una muestra de estudio formada por estudiantes y egresados universitarios de distintas universidades españolas. Así, se caracterizan los resultados obtenidos en el conjunto de la muestra y, posteriormente, dividiendo la muestra en los subgrupos de participantes y no participantes en el Programa Erasmus. De los datos recolectados se concluye que los jóvenes universitarios españoles que forman la muestra de estudio, independientemente de su participación en el Programa Erasmus, son ciudadanos globales insertos en la esfera pública. Ahora bien, en las percepciones de los estudiantes Erasmus se reconoce una influencia positiva sobre el sentimiento europeo y el interés por la Unión Europea, así como por la confianza en las instituciones europeas. En cambio, los Erasmus tienden a ser más críticos en otros aspectos.
\end{abstract}

\section{Palabras clave}

Opinión pública, Unión Europea, viaje, identidad, actitud política, estudiante universitario.

Forma sugerida de citar: Fernández-Rovira, Cristina (2019). El viaje como elemento performativo de la esfera pública europea. El caso del Programa Erasmus. Universitas, 30, pp. 79-95. 


\begin{abstract}
The article combines the travel experience, the construction of identity and the European public sphere through the Erasmus Programme. The topic is interesting and original in order to know how the different variables interrelate in this vital experience. The study describes the attitudes and perceptions of Spanish students about the construction of their own European identity and the common public sphere under a comparative approach between those who have participated in the Erasmus Programme and those who have not. The core of the analysis is the data obtained through a closed questionnaire applied to a sample made up of students and university graduates from different Spanish universities. Thus, the article characterizes the results obtained in the whole of the sample and, subsequently, it offers an analysis of the subgroups of participants and non-participants in the Erasmus Programme. The article concludes that the Spanish university students, regardless of their participation in the Erasmus Programme, are global citizens inserted in a transnational public sphere, who frequently show interest in European news. However, the perceptions of the participants are more positive towards European feelings. They show more interest in the European Union, as well as more confidence in the European institutions. In contrast, Erasmus students tend to be more critical in other aspects.
\end{abstract}

Keywords

Public opinion, European Union, travel, identity, political attitude, university student.

\title{
Introducción y estado de la cuestión
}

El viaje ha sido a lo largo de la historia un elemento clave en el desarrollo de las sociedades. Desde las migraciones de los primeros homínidos hasta la configuración del mundo globalizado, la humanidad ha usado el viaje como pieza fundamental para configurar su cosmovisión y su personalidad. En la actualidad, las distintas identidades están atravesadas en mayor o menor medida por la experiencia del viaje, bien sea en forma de migración, de turismo o de descubrimiento.

La sociedad globalizada actual —entendida la globalización como un fenómeno que supera las definiciones ofrecidas por la Comisión Europea (2002) y el Banco Mundial (2002), que ven el fenómeno como una tendencia a una mayor integración e interdependencia entre los países y las regiones del planeta y como el hecho de que las actividades económicas que más rápidamente han crecido son aquellas que tienen lugar entre los países, respectivamente- tiene en el viaje un elemento capital. Siguiendo la definición 
de globalización propuesta por Held y McGrew (2000), la integración global se sale del ámbito económico, ya que se refiere al conjunto de procesos interrelacionados que operan en los dominios primarios del poder social, político y cultural. Si se admite esto último, la globalización va más allá del intercambio de mercancías, capitales y servicios y promueve una cultura global, auspiciada por medios de comunicación planetarios.

La industrialización de las sociedades occidentales permitió el desarrollo de medios de comunicación y de transporte cada vez más eficaces. Se hizo posible el traslado rápido y económico de gran cantidad de mercancías, pero también de seres humanos. Ello supuso el inicio de una nueva mentalidad, a la que contribuyeron primero los periódicos, en el inicio del siglo XX, y la radio a partir de los años cuarenta. Más tarde, en las sociedades del bienestar, la televisión ejerció una gran influencia en la configuración de los estilos de vida, en los que el turismo se empezaba a popularizar. Así se llegó a la era de internet y de la sociedad de la información y, con ellos, a una suerte de sujeto global. Este proceso influye en la configuración de las identidades personales y sociales, así como en la existencia de una esfera pública globalizada.

Para García (2016), los devaneos contemporáneos de la identidad personal hacen que experimentemos un modo de vida que oscila de los vínculos adscriptivos a los electivos, lo que trastoca el sentido de la identidad personal. Tal y como la entiende Castells (1999):

Identidad, en términos sociológicos, es el proceso por el cual los actores sociales construyen el sentido de su acción atendiendo a un atributo cultural (o conjunto articulado de atributos culturales) al que se da prioridad sobre otras fuentes posibles de sentido de la acción. Puede darse el caso de varias identidades en un individuo, pero tal pluralidad es siempre fuente de tensión. (p. 7).

Sobre un mundo de identidades insiste Touraine (1998), al considerar que en realidad la globalización no existe, sino que se trata de una construcción ideológica. La tensión de la sociedad contemporánea entro lo global y lo local cuestiona qué tipo de identidad se está forjando. Según Bolívar (2001), se debe explorar la tensión entre la sociedad red y el yo-nosotros identitario, entre los valores cívicos y las diferencias culturales.

Partiendo de la premisa de la existencia del ciudadano globalizado, es decir, aquella persona que goza de un estatus de ciudadano de un Estado que lo faculta para ejercer sus deberes y derechos, pero que está inserto en la so- 
ciedad de la información global, se infiere la existencia de una esfera pública que igualmente traspasa los límites del Estado-nación. El concepto de esfera pública se entiende como el lugar de la vida social en el que se puede crear opinión pública, con acceso garantizado a todos los ciudadanos (Habermas, Lennox, \& Lennox, 1974). La aldea global (McLuhan, 1962) y la esfera pública global (Habermas, 1962) empiezan a formar parte de la cotidianidad de la clase media a medida que la tecnología avanza. El mundo occidental está cada vez más conectado y, en especial, la integración europea configura un espacio común en el que hay más de 500 millones de personas.

\section{La especificidad de la Unión Europea (UE) Hacia la esfera pública común}

En Europa, el Tratado de Roma (1957) inició la Comunidad Económica Europea. Transformado en Unión Europea, el proyecto ha continuado hasta el día de hoy. Es precisamente aquí donde Habermas (1962) sitúa el origen de la esfera pública, en la burguesía europea de los siglos XVIII y XIX y la define como un lugar de debate y discusión que se ve transformado con la llegada de los medios de comunicación masivos en el siglo XX. En los últimos años, la Comisión Europea ha promovido medidas para priorizar la esfera pública específicamente europea, como las iniciativas Plan D for Democracy, Dialogue and Debate (CEC, 2005) y White Paper on a European Communication Policy (CEC, 2006). Se entiende que la existencia de una esfera pública es importante como precondición para la realización de la soberanía popular, ya que permite opinar a todo el mundo sin limitaciones (Eriksen, 2004).

De hecho, siguiendo a Calhoun (2003), la esfera pública facilita la elección colectiva y permite la producción, reproducción y transformación del imaginario social, además de ser un medio de integración social, una forma de solidaridad social y una arena para debatir. El cuestionamiento radica en si la esfera pública puede establecerse más allá de las fronteras del Estadonación (Bellamy, \& Castiglione, 2001; Calhoum, 2003; Eriksen, \& Fossum, 2002), ya que en general el consenso se da en que las esferas nacionales no pueden trasponerse a nivel europeo (Castells, 1997). Sin embargo, para Bee y Bozzini (2010), el ambiente europeo en Bruselas conduce al establecimiento real de una esfera pública transnacional. 
Existe un debate abierto acerca de la existencia de una esfera pública europea, sobre quién la conforma y cómo se desarrolla. Contrariamente a la hipótesis del déficit de esfera pública europea, Koopmans y Erbe (2004) comprobaron que los medios de comunicación alemanes reflejaban la europeización del policy-making. Por otro lado, Koopmans (2007) concluyó que los actores gubernamentales y ejecutivos son, con mucho, los beneficiarios más importantes de la europeización de los debates públicos en comparación con los actores legislativos y partidistas, y aún más en comparación con los actores de la sociedad civil, que están extremadamente poco representados en los debates públicos europeos. Según Dahlgren (2006), la esfera pública no empieza y acaba cuando el contenido mediático llega a la audiencia; eso es un eslabón en las largas cadenas comunicativas y culturales que incluyen cómo el contenido mediático es recibido, entendido y usado por los ciudadanos. Por tanto, la esfera pública, de un modo amplio, integra todo el proceso comunicativo y, por ende, cultural.

El problema en la Unión Europea radica en que se trata de un experimento de integración único en el mundo por el nivel de soberanía compartida alcanzado, en el que se discute si existe una identidad colectiva capaz de crear una esfera pública común. A este respecto, Hepp et al. (2016) concluyeron que lejos de acusar la fragmentación de la identidad europea, la lucha colectiva por entender la reciente crisis del euro ayudó a cimentar una identidad común, señalando que existen más cosas que unen a los europeos de las que los separan. La necesidad de reconciliar multiculturalismo, ciudadanía e identidad colectiva es señalada por Delgado-Moreira (2017), al indicar que existen políticas europeas sobre la ciudadanía multicultural y que las instituciones de la UE elaboran medidas para crear identidad y ciudadanía europea. Sin embargo, alerta de que existe una falta de conexión sustantiva entre la ciudadanía europea y la identidad de la Unión. Eriksen (2005) argumenta que la cooperación europea y la resolución de problemas crean espacios públicos, pero hasta el momento no han producido una sola esfera pública europea general. Más bien, lo que uno encuentra son públicos segmentados, transnacionales, que evolucionan en torno a redes de políticas constituidas por el interés común en ciertos campos de políticas.

Cabe resaltar, siguiendo las aportaciones de Scammell y Semetko (2018), que actualmente se ha perdido la hegemonía de la vida pública estructurada por el Estado y vinculada territorialmente por la radio, la televisión, los periódicos y los libros y que la multiplicidad de espacios de comunicación en 
red alejan la idea de una esfera pública unificada. En su lugar, lo que se dan son esferas públicas de diferentes tamaños, superpuestas e interconectadas.

\section{El viaje mediado por el Programa Erasmus}

El Programa Erasmus nació en 1987 con la voluntad de fomentar el intercambio académico y cultural entre universitarios de distintos centros de formación del espacio económico europeo, más Suiza y Turquía. En el núcleo de la experiencia está el viaje a otro país de la Unión Europea y la posibilidad de vivir y estudiar en un entorno distinto al del país de origen entre tres y doce meses, con la garantía de que los estudios cursados van a ser reconocidos en la universidad de origen. En 2014, el Programa Erasmus se transformó en Erasmus+ dentro de la Estrategia Europea 2020 (Jones, 2017; Hubble, Bellis, \& Bolton, 2018).

Erasmus+ recoge los proyectos anteriores y se ha convertido en el programa de la Unión Europea que apoya la educación, la formación, la juventud y el deporte en el continente. Se extiende hasta 2020 y tiene un presupuesto de 14700 millones. Su principal objetivo es ofrecer oportunidades de estudio, adquisición de experiencia y voluntariado a cuatro millones de ciudadanos europeos. Así, no se dirige solamente a estudiantes, sino que también pueden beneficiarse docentes, practicantes u organizaciones, entre otros.

En este artículo se ha considerado el Programa Erasmus y la variante Erasmus+ en lo que concierne solo a estudiantes universitarios y egresados. Se parte de la base que, más allá del contenido académico, el viaje paneuropeo mediado por el Programa Erasmus (que conlleva ventajas académicas y económicas) influye en la cosmovisión de los participantes acerca de la construcción de la identidad europea y de la configuración de la esfera pública europea, puesto que amplía los horizontes cognitivos de los participantes, ya de por sí insertos en el mundo global. Además, el programa se relaciona con la internacionalización de la educación superior más allá de la movilidad europea (Haug, 2016; Villalón de la Isla, 2017).

La investigación pretende explorar el papel del Programa Erasmus en relación con el viaje como elemento performativo de la esfera pública europea. Por ello, además de la revisión bibliográfica, se ha realizado una encuesta a estudiantes universitarios y egresados de distintas universidades españolas.

El objetivo principal del análisis es observar y describir en qué medida el viaje mediado por el Programa Erasmus contribuye a modificar las per- 
cepciones ciudadanas sobre la esfera pública europea en las personas que han tenido oportunidad de participar en él en comparación con los que no han participado en el programa. Del mismo modo, también se pretende caracterizar cómo la participación en el programa altera las percepciones sobre la identidad europea.

El estudio parte de la hipótesis de que el viaje como experiencia principal del Programa Erasmus, más allá de su contenido académico, favorece la creación de la esfera pública europea e influye positivamente en la percepción ciudadana sobre la Unión Europea en España. Del mismo modo, se considera que las capas más jóvenes de la sociedad española con estudios universitarios están insertas en una incipiente esfera pública europea. Además, se trata de ciudadanos globales y totalmente integrados en la sociedad digital.

La investigación relaciona de manera descriptiva y compara las percepciones acerca del fenómeno entre los subgrupos que conforman la muestra de análisis.

\section{Material y métodos}

El estudio se ha llevado a cabo bajo dos técnicas de investigación: la revisión bibliográfica y el cuestionario. La revisión de la literatura ha servido para refinar los objetivos de la investigación y para la posterior elaboración de las preguntas que forman la encuesta. Así, el cuestionario contiene veinte preguntas encaminadas a recolectar información relevante para conocer los conceptos que se relacionan en la investigación. Se trata de preguntas cerradas.

La muestra se ha acotado a estudiantes y egresados universitarios por ser los únicos que hasta fechas recientes han tenido la posibilidad de participar en el Programa Erasmus. Por tanto, se trata de una muestra no probabilística, ya que su elección viene dada por las características de la investigación (Hernández, Fernández-Collado, \& Baptista, 2006). El total de la muestra está formado por 124 respuestas válidas.

El $100 \%$ de la muestra está formada por estudiantes o exestudiantes de universidades españoles, de los cuales el 66,1\% son mujeres y el 33,9\% son hombres. El 96\% de los encuestados se sitúa entre los 18 y los 30 años, y el 4\% tiene más de treinta años, lo que indica que todos han podido participar en el programa creado en 1987. De ellos, el 95,2\% manifiesta conocer el Programa Erasmus. Sin embargo, solo el 24,2\% ha participado en la experiencia paneuropea. 
El método comparativo resulta eficaz para poder describir el objeto y comprobar la hipótesis planteada, puesto que permite poner en relación las actitudes recogidas en los distintos datos para los dos subgrupos.

\section{Análisis y resultados}

De los datos obtenidos se observa que los universitarios españoles viven en un mundo globalizado, con facilidad para viajar o realizar transacciones internacionalmente, en especial con la Unión Europea. El ciudadano global se refleja en la muestra de estudio, ya que el 36,3\% asegura viajar al extranjero dos o tres veces al año; el 32,3\% dice hacerlo una vez al año; el 14,5\%, más de tres veces al año; el 12,9\%, manifiesta que viaja fuera de España una vez cada dos o tres años; y, tan solo el $4 \%$ dice no hacerlo nunca. Por tanto, el $68,6 \%$ de la muestra viaja al extranjero entre una y tres veces al año. La experiencia del viaje y su facilidad en el mundo de hoy se pone de manifiesto. Ello se refuerza con el hecho de vivir integrado en la Unión Europea, el único territorio en el mundo en el que 28 Estados tienen grandes dosis de políticas comunes y en el que se comparte una esfera pública.

Así, el 31,5\% de las personas asegura que alguna vez realiza actividades con otro país de la UE, mientras el 27,4\% dice no hacerlo muy frecuentemente. En cambio, el 21,8\% asegura que lo hace frecuentemente y el 7,3\%, muy frecuentemente, frente al $12,1 \%$ que no lo hace nunca. Del total de la muestra, sin embargo, casi la mitad, el 49,2\% asegura que se mantiene frecuentemente informado de lo que pasa en la Unión Europea y el 7,3\% dice que lo hace muy frecuentemente. Al contrario, el 29\% dice no mantenerse informado muy frecuentemente sobre asuntos europeos; el 13,7\% dice que lo hace alguna vez y el $0,8 \%$, nunca.

De los datos anteriores se desprende que el 87,9\% mantiene relaciones con otros países de la UE, aunque puedan ser esporádicas y de distinta naturaleza, como personales, comerciales o de negocios. Un 23,4\% de los encuestados ha trabajado o vivido en otro país de la UE distinto a España durante tres meses o más; pero, el 49,2\% tiene algún familiar que sí ha vivido o trabajado en otro Estado miembro. De ello se extrae que la Unión Europea está presente en el imaginario colectivo de, al menos, la mitad de la muestra. A la vez, queda claro que los encuestados se interesan por la actualidad, ya que la totalidad de la muestra consulta medios de información a diario (71\%), 
semanalmente $(21 \%)$ o alguna vez al mes $(8,1 \%)$. Además, el $63,7 \%$ opina que la UE se presenta en los medios con aspectos positivos y negativos; el $31 \%$ cree que solo se presenta como algo bueno y el 4,8\% como algo malo.

El 60,5\% de la muestra mantiene una opinión favorable a que España pertenezca a la Unión Europea, lo que coincide con el tradicional europeísmo español mostrado en distintos sondeos; frente al 34,7\% que es indiferente y el $4,8 \%$, que se muestra desfavorable. El $37,9 \%$ de los encuestados se siente igual de europeo que de su comunidad autónoma y el 30,6\%, igual de europeo que de español, frente al 24,2\% que no se siente europeo y el 7,3\% que solamente se siente europeo. Preguntados por su entorno, los encuestados aseguran que entre sus familiares y amigos los sentimientos hacia la UE no son favorables ni desfavorables $(58,1 \%)$, frente al $27,4 \%$ que los considera favorables y el 4,8\%, muy favorables. En cambio, el $8,9 \%$ los ve desfavorables. De ello se desprende que el contexto relacional acerca la UE es más bien indiferente en la muestra de estudio.

De igual modo, del total de la muestra, el $42 \%$ de los encuestados se considera indiferente acerca de si la pertenencia a la UE le ha beneficiado, frente al 38\% que considera que sí lo ha hecho. En cambio, el 53\% está de acuerdo en que España ha salido beneficiada de formar parte de la UE, mientras que el $16 \%$ se muestra indiferente, igual que el $16 \%$ que se muestra muy de acuerdo. Al contrario, el $12 \%$ de los preguntados está en desacuerdo y considera que España no ha sido beneficiada de la integración europea.

Sobre si la Unión Europea se entromete en los asuntos políticos españoles, el 29\% de la muestra es indiferente, el $25 \%$ está de acuerdo y el $22 \%$, en desacuerdo. La indiferencia gana también entre los encuestados al ser preguntados sobre si, en general, lo que es bueno para la UE es bueno para España, con el 37\% que no está ni en acuerdo ni en desacuerdo, frente al 29\% que está en desacuerdo y el 19\%, que está de acuerdo. De hecho, casi la mitad de la muestra, el 49\%, no siente ni confianza ni desconfianza hacia las instituciones europeas, mientras que el $21 \%$ siente bastante confianza y el $16 \%$ manifiesta sentir desconfianza.

Los sentimientos de desconfianza difieren cuando se trata de las instituciones estatales, según los datos recolectados. De este modo, el $49 \%$ de la muestra siente mucha desconfianza hacia el Gobierno español, el 27\% siente desconfianza y el $16 \%$ se muestra indiferente. Por lo que se refiere al gobierno autonómico, los niveles extremos de desconfianza no son tan elevados, ya que es el $19 \%$ de la muestra el que siente mucha desconfianza. El 
$31 \%$ de la muestra siente desconfianza por el gobierno autonómico, el 29\% es indiferente y, de nuevo, el 19\% dice sentir bastante confianza. Por otro lado, la mayoría de la muestra de estudio, el $81 \%$ siente mucha desconfianza por la monarquía.

De ello se atisba que las instituciones estatales generan opiniones más radicalizadas que las europeas.

La comparativa de los subgrupos permite observar cómo la experiencia del viaje influye en aquellos que han realizado un Erasmus. Sobre la existencia de una esfera pública europea, se ve que aquellos que se mantienen muy frecuentemente informados sobre la UE son el $17 \%$ entre los que han realizado un Erasmus y son el 4\% entre los que no lo han hecho. Igualmente, entre los participantes del programa, el $3 \%$ dice mantenerse informado alguna vez, frente al $17 \%$ que dice hacerlo alguna vez entre los que no han participado en el programa europeo. Con ello se infiere que aquellas personas que han participado en la experiencia europea se muestran más frecuentemente interesadas en los asuntos informativos que conciernen a la UE, lo que incide en reforzar la esfera pública europea. Entre aquellos que han participado en el programa se aprecian posiciones más críticas al respecto de cómo se presenta la Unión Europea en los medios de comunicación, ya que el 37\% de los participantes considera que se presenta a la Unión como algo bueno, frente al 30\% que lo considera entre los no participantes; el 53\% de los participantes piensa que se muestra como algo con aspectos positivos y negativos, mientras que el $67 \%$ de entre los no participantes es el que lo considera así; pero, el $10 \%$ de los participantes observa que se presenta como algo malo, frente al 3\% que lo percibe entre los no participantes en el Erasmus.

En lo referente a la construcción de la propia identidad, entre aquellas personas que han formado parte del Programa Erasmus está más extendido el sentimiento de ser solamente europeo, ya que el $17 \%$ de ellos dice sentirse así, mientras que el $4 \%$ de los que no han participado en el programa aseguran sentirse solamente europeos. La experiencia del viaje mediada por el Programa Erasmus influye en la configuración de la propia identidad, sobre todo si se contrapone a la opinión acerca de la pertenencia de España a la UE que ofrece datos similares tanto en los participantes como entre los no participantes en el programa, en que más de la mitad son favorables a la integración.

Las diferentes percepciones acerca de la Unión Europea se hacen más palpables entre los que han hecho algún Erasmus y los que no al opinar sobre si pertenecer a la Unión les ha beneficiado personalmente, pues el 27\% 
entre los que han participado están muy de acuerdo en que les ha beneficiado, frente al 9\% de entre los que no han participado. Igualmente, entre los participantes, el $43 \%$ está de acuerdo, frente al $36 \%$ de los no participantes. La indiferencia cae entre aquellos que han vivido la experiencia del viaje Erasmus, ya que el $27 \%$ no está en acuerdo ni en desacuerdo en que les haya beneficiado, mientras el $47 \%$ de los no participantes se muestra indiferente ante esta cuestión.

En cuanto a la opinión sobre si España ha salido beneficiada de su pertenencia a la Unión Europea se pone de manifiesto que los que han realizado un Erasmus están muy de acuerdo (30\%) frente a los que lo están entre los que no lo han realizado (12\%). En sentido contrario, los que están en desacuerdo son el 3\% entre los Erasmus y el 14\% entre los que no han hecho Erasmus. Se observa que los participantes ven más ventajas que los no participantes en pertenecer a la Unión Europea. Ello se refuerza con la percepción del $34 \%$ de los participantes que se muestra indiferente acerca de si la UE se entromete en los asuntos políticos españoles y el $23 \%$ que se muestra muy en desacuerdo, frente al $28 \%$ que es indiferente y el $14 \%$ que está muy en desacuerdo entre los no participantes. De acuerdo con la afirmación está el 20\% de los participantes y el $26 \%$ de los no participantes.

Las divergencias entre los subgrupos se ponen de manifiesto al considerar si lo que es bueno para la Unión Europea es también bueno para España, ya que el $10 \%$ de los participantes en el Programa Erasmus está de acuerdo, frente al $21 \%$ de los que no ha participado lo está. En desacuerdo está el $40 \%$ de los que han realizado un Erasmus, mientras que el 26\% de los que no lo ha hecho está en desacuerdo.

La discrepancia entre los participantes y no los participantes se hace evidente también en las percepciones de la desconfianza ciudadana hacia distintas instituciones de la democracia representativa de distintos niveles de gobernanza, tal y como muestran los datos recogidos.

La percepción de la confianza es diferente entre ambos subgrupos con respecto a las instituciones comunitarias, como era previsible. Sin embargo, también se aprecian diferencias en la percepción de la confianza hacia los gobiernos estatal y autonómico entre los subgrupos. En cambio, esto no ocurre con la percepción de la confianza hacia la monarquía española, en la que prevalece la desconfianza mayoritariamente en los dos subgrupos. Así pues, se infiere que otros aspectos y no solo la participación en el Programa Erasmus pueden influir en la confianza hacia las distintas instituciones. 
Resulta destacable que el $40 \%$ de los que han realizado un Erasmus no siente ni confianza ni desconfianza hacia el Europarlamento, la Comisión y el Consejo Europeo. Aun así, esa indiferencia es mayor entre los que no han participado en un Erasmus, con el 52\%. Por el contrario, son más numerosos (33\%) aquellos que habiendo realizado un Erasmus sienten bastante confianza en las instituciones europeas, frente al $17 \%$ que no habiéndolo realizado sienten bastante confianza. De manera similar pero en sentido inverso, se observa que desconfían más los que no han hecho un Erasmus (19\%) frente al $7 \%$ que sí lo ha realizado y que dice que desconfía. Paradójicamente, aquellos que han participado en el Programa Erasmus muestran una posición más extrema de desconfianza hacia las instituciones europeas, frente aquellos que no han participado en el programa. De este modo, el $17 \%$ de los participantes asegura sentir mucha desconfianza, mientras que es el 7\% el que lo asegura en el caso de los no participantes.

Precisamente, es en el caso de la desconfianza y del grado de mucha desconfianza hacia el Gobierno de España donde también se pone de manifiesto más diferencia entre los dos subgrupos. Entre los que han realizado Erasmus, la opción mayoritaria (40\%) es la de mucha desconfianza hacia el Gobierno español, 12 puntos inferior que esa opción (52\%) entre los que no han realizado un Erasmus. Sin embargo, la opción de desconfianza es mayor (33\%) entre los que han participado en un Erasmus que entre los que no han participado, ya que un $26 \%$ de ellos se sitúa en la desconfianza hacia el Gobierno estatal. En lo que tiene que ver con el Gobierno autonómico, los datos aparecen más dispersos. En los dos subgrupos se dan valores similares entre aquellos que se inclinan por la mucha desconfianza $(20 \%$ y $19 \%$ entre los participantes y los no participantes). En cambio, hay 8 puntos de diferencia entre los que sienten bastante confianza hacia el Gobierno autonómico, entre el 13\% de los participantes y el $21 \%$ de los no participantes que se ubican en esa opción. En cuanto a la posición de no sentir ni confianza ni desconfianza se da una diferencia de 6 puntos entre las opiniones de los subgrupos, del 34\% de los participantes al $28 \%$ de los no participantes en el Programa Erasmus.

De los datos anteriores se desprende que, excepto en el caso del grado de mucha desconfianza en el Gobierno español, las personas que ha realizado un Erasmus o bien muestran mayor indiferencia o bien tienen opiniones más tendentes a la desconfianza hacia los gobiernos estatal y autonómico.

Entre aquellas personas de la muestra que han participado en el Programa Erasmus se observa que en la mayoría de casos esa participación ha ge- 
nerado un mayor interés por la Unión Europea, ha permitido establecer un grupo de amigos internacionales y consideran que les ha beneficiado académica y laboralmente. En contraste, menos personas creen que realizar un Erasmus les ha servido para interiorizar los valores europeos.

Así, el 64\% de las personas consideran que realizar un Erasmus ha hecho que su interés por la Unión Europea crezca, el 17\% de los que han participado en el programa está muy de acuerdo con ello y el $43 \%$ está de acuerdo. El 27\% de ellos no se muestra ni en acuerdo ni en desacuerdo, y el 13\% está en desacuerdo (3\%) y muy en desacuerdo (10\%). El interés despertado por la realización del viaje es un factor coadyuvante en el fortalecimiento de la esfera pública europea. De la misma manera, mantener un entorno personal de relaciones con otras personas de los Estados miembros es también un factor que ayuda a la construcción de la esfera pública europea, como lo expresa el $63 \%$ de las personas que han participado en el programa al estar muy de acuerdo en que les ha permitido tener un grupo de amigos internacional y el $20 \%$, que se muestra de acuerdo con ello. Por tanto, el $83 \%$ de los que han realizado un Erasmus valora positivamente esa posibilidad, que unida al interés por la comunidad europea puede generar más inclinación hacia la existencia de la esfera pública común. En el plano personal, el $87 \%$ de los encuestados con Erasmus se muestra favorable a los efectos del programa en su currículum. Entre los preguntados, el 47\% está muy de acuerdo y el $40 \%$ está de acuerdo en que les ha beneficiado académica y laboralmente, frente al $13 \%$ que se muestra indiferente.

Por lo que se refiere al conocimiento de la UE gracias al Programa Erasmus o al fortalecimiento de la propia identidad europea, la opción mayoritaria es la de la indiferencia, mientras que en cuanto a interiorizar los valores europeos, la indiferencia y el desacuerdo empatan. De esta forma, el 33\% de las personas que han realizado un Erasmus se muestran indiferentes acerca de si el programa ha contribuido en reforzar su identidad europea, aunque los que están muy de acuerdo (17\%) y de acuerdo (30\%) suman el 47\%, son, por tanto, más numerosos. En cambio, los que están en desacuerdo (17\%) y muy en desacuerdo (13\%) son el 30\% de los encuestados. Similarmente, a si participar en el Programa Erasmus contribuye a conocer mejor el funcionamiento de la UE, el $34 \%$ se muestra indiferente, pero la suma de los que están muy de acuerdo (10\%) y de acuerdo (30\%) resulta mayor, con un $40 \%$. Al contrario, el $23 \%$ se muestra en desacuerdo y el 3\%, muy en desacuerdo.

En el caso de la utilidad del programa para interiorizar los valores europeos, se da un empate con el $27 \%$ de los que se muestran indiferentes y el 
$27 \%$ de los que están en desacuerdo. Aun así, si se une al grado de desacuerdo el de muy en desacuerdo (13\%) se obtiene un resultado del 40\%; por tanto, es mayor que el de los que manifiestan una opinión de muy de acuerdo (13\%) y de acuerdo (20\%), cuya suma da el $33 \%$. En ese punto se puede ver un debilitamiento en cuanto a la homogeneización de la construcción de la identidad europea a través del Programa Erasmus.

\section{Discusión y conclusiones}

La hipótesis de que la experiencia del viaje mediada por el Programa Erasmus favorece las opiniones sobre la Unión Europea queda demostrada a la luz de los datos obtenidos y está en línea con otros estudios que resaltan el éxito del programa (Cairns, 2017; Santos, \& Cunha, 2018). Del mismo modo, la experiencia también refuerza la existencia de la esfera pública europea en términos informativos. Ello se desprende de la perspectiva comparada entre aquellos que han formado parte del programa durante su etapa universitaria y aquellos que no; así como al considerar sus opiniones acerca de la experiencia Erasmus.

El sentimiento europeo en la propia identidad está más extendido entre las personas que han hecho un Erasmus, quienes también consideran que España ha salido beneficiada de pertenecer a la UE, así como piensan que también les ha beneficiado a nivel personal en lo laboral y académico. Además, casi la mitad de los que han participado en un Erasmus piensan que el viaje ha contribuido a reforzar su identidad europea, igual que un $40 \%$ considera que gracias a esa experiencia conoce mejor el funcionamiento de la UE. En cambio, un número mayor de participantes considera que la experiencia no ha servido para interiorizar los valores europeos, algo que se muestra como un punto débil en la construcción de la propia identidad europea, ya que los participantes no se muestran conscientes de haber desarrollado los valores cosmopolitas asociados a la UE.

Según los datos encontrados, el viaje Erasmus influye en la percepción de confianza en las instituciones europeas, ya que el $40 \%$ de los que han realizado Erasmus no sienten confianza ni desconfianza en el Europarlamento, la Comisión y el Consejo Europeo, pero son más los indiferentes hacia ellas que no han hecho Erasmus (52\%). Sin embargo, aunque la posición más radical de desconfianza es mayor entre los Erasmus, también son más los que sienten bastante confianza y menos los que desconfían. 
Queda fuera del alcance del estudio determinar si existe una esfera pública europea homogénea o una multiplicidad de ellas superpuestas e interconectadas, pero lo que sí demuestran los datos obtenidos es que los jóvenes universitarios están insertos en una esfera pública transnacional. Independientemente de la participación en el programa, la UE está en la cosmovisión de los encuestados, como lo demuestra que el $87 \%$ mantenga alguna relación con la comunidad supranacional. Más del $60 \%$ del total de los encuestados es favorable a la pertenencia de España a la UE, casi la mitad se mantiene informada de los asuntos europeos y tiene algún familiar que ha vivido o trabajado en otro país miembro. Eso sí, más de la mitad de aquellos que han hecho un Erasmus se interesan más por la UE y han podido mantener una red de amigos internacionales. Además, los participantes se mantienen más frecuentemente informados sobre asuntos comunitarios que los no participantes, lo que refuerza la existencia de una esfera pública comunitaria.

\section{Bibliografía}

Bee, C., \& Bozzini,E. (2010). Mapping de European Public Sphere.London: Routledge. Bellamy, R., \& Castiglione, D. (2001). The Uses of Democracy: Reflections on the European Democratic Deficit. En E.O. Eriksen y J.E. Fossum, Democracy in the EU: Integration through Deliberation (pp. 65-84). London: Routledge.

Bolívar, A. (2001). Globalización e identidades:(des) territorialización de la cultura. Revista de educación, 1, 265-288.

Cairns, D. (2017). The Erasmus undergraduate exchange programme: a highly qualified success story? Children's Geographies, 15(6), 728-740.

Calhoun, C. (2003). The Democratic Integration of Europe: Interests, Identity, and the Public Sphere. En M. Berezin y M. Schain, Europe without Borders: Remapping Territory, Citizenship, and Identity in a Transnational Age. Baltimore: The Johns Hopkins University Press.

Castells, M. (1997). The Power of Identity. The Information Age: Economy, Society and Culture Vol. II. Oxford: Blackwell.

Castells, M. (1999). Globalización, identidad y estado en América Latina. Santiago de Chile: PNUD, 1-18.

Comission of the European Comunities (2005). Plan-D for Democracy, Dialogue and Debate. Bruselas: CEC.

Comission of the European Comunities (2006). Paper on a European Communicaton Policy. Bruselas: CEC. 
Dahlgren, P. (2006). Doing citizenship: The cultural origins of civic agency in the public sphere. European Journal of Cultural Studies, 9(3), 267-286.

Delgado-Moreira, J. M. (2017). Multicultural Citizenship of the European Union. London: Routledge.

Eriksen, E. O. (2004). Conceptualizing European Public Spheres. General, Segmented, and Strong Publics. ARENA Working Paper 3/04.

Eriksen, E. O. (2005). An Emerging European Public Sphere. European Journal of Social Theory, 8(3), 341-363.

Eriksen, E.O., \& Fossum, J.E. (2002). Democracy in the European Union: Integration Through Deliberation? London: Routledge.

European Commission (2002). Responses to the challenges of globalization. A study on the international monetary and financial system and of financing for globalization. European Economy, (Special Report 1).

García, B. (2016). La constitución de la identidad personal en el nuevo capitalismo y sus repercusiones morales. Daimon. Revista Internacional de Filosofía, 67, 117-131.

Habermas, J. (1962). [1989]. The Structural Transformation of the Public Sphere. Massachusetts: The MIT Press.

Habermas, J., Lennox, S., \& Lennox, F. (1974). The Public Sphere: An Encyclopedia Article (1964). New German Critique, (3), 49-55.

Haug, G. (2016). La internacionalización de la educación superior: más allá de la movilidad europea. La cuestión universitaria, (6), 20-29.

Held, D., \& McGrew A. G. (2000). The Global Transformations reader: An Introduction to the Globalization Debate. Massachusetts: Polity Press.

Hepp, A., Elsler, M., Lingenberg, S., Mollen, A., Möller, J., Offerhaus, A., Sword, K., \& Pospielovsky, D.V. (2016). The Communicative Construction of Europe: Cultures of Political Discourse, Public Sphere and the Euro Crisis. Palgrave MacMillan.

Hernández, R., Fernández-Collado, C., \& Baptista, P. (2006). Metodología de la investigación. México: McGraw Hill.

Hubble, S., Bellis, A., \& Bolton, P. (2018). The Erasmus programme. Briefing paper, House of Commons.

Jones, H. C. (2017). Celebrating 30 years of the Erasmus programme. European Journal of Education, 52(4), 558-562.

Koopmans, R., \& Erbe, J. (2004). Towards a European public sphere? Vertical and horizontal dimensions of Europeanized political communication. Innovation: The European Journal of Social Science Research, 17(2), 97-118. 
Koopmans, R. (2007). Who inhabits the European public sphere? Winners and losers, supporters and opponents in Europeanised political debates. European Journal of Political Research, 46(2), 183-210.

McLuhan, M. (1962). The Gutenberg Galaxy. Canada: University of Toronto Press.

Santos, Y., \& Cunha, A. (2018). The participation of Portuguese students in Erasmus: From its European conception to its implementation in universities. Estudos do Século, XX(18), 97-113.

Scammell, M., \& Semetko, H. (2018). The Media, Journalism and Democracy. London: Routledge.

Touraine, A. (1998). Sociedad política, democracia y responsabilidad individual. Intervención en el encuentro en Rabat (3-4, abril) sobre globalización.

Villalón de la Isla, E. M. (2017). La movilidad estudiantil en el proceso de internacionalización. Estrategias metodológicas para su estudio. Revista Española de Educación Comparada, (29), 297-314.

Fecha de recepción: 2018/I1/12; Fecha de aceptación: 2019/02/I2;

Fecha de publicación: 2019/03/01 